\title{
Features and Outcome of Hepatobiliary Tuberculosis among Filipino Children: Report of a Six-year Experience
}

\author{
Evangeline P. Capul, Germana Emerita V. Gregorio and Ma. Liza Antoinette M. Gonzales \\ Department of Pediatrics, College of Medicine and Philippine General Hospital, University of the Philippines Manila
}

\begin{abstract}
Background. The features and outcome of hepatobiliary tuberculosis (HBTB) have not been extensively reported in children.

Objective. To describe the clinical, biochemical, radiologic, microbiologic and histologic features and outcome of children diagnosed with HBTB.

Methods. Data of HBTB patients aged 0-18 years were collected by review of medical records and as they were admitted. Cases were classified as bacteriologically-confirmed (positive AFB smear, TB culture or PCR of bile/liver tissue) or clinically-diagnosed (clinical, histologic and/or radiologic evidence).

Results. A total of 36 patients were included (mean age: 13yrs; $64 \%$ males): three bacteriologically-confirmed and 33 clinically-diagnosed. Most common signs/symptoms were weight loss (69\%), fever (67\%), hepatomegaly (61\%) and jaundice (53\%). Of the total, $68 \%$ had hypoalbuminemia, $50 \%$ increased transaminases and $47 \%$ prolonged prothrombin time. Fifteen (42\%) patients were AFB positive on various microbiologic specimens. Most common imaging finding was hepatic calcification (64\%). Of 11 patients with liver biopsy, seven (64\%) had chronic/ granulomatous inflammation. All 36 were managed medically. Eight were lost to follow up, six died, and $22(61 \%)$ are alive, nine with complete resolution of liver disease.
\end{abstract}

Conclusion. Hepatobiliary tuberculosis presents with non-specific clinical and biochemical findings. Several investigations are necessary to confirm the diagnosis. Overall response to anti-TB treatment is satisfactory with possible resolution of liver disease.

Key Words: hepatobiliary TB, hepatic granuloma, hepatic calcification, AFB smear, TB PCR

\section{INTRODUCTION}

Paper presented at the $24^{\text {th }}$ Annual Convention of the Pediatric Infectious Disease Society of the Philippines, February 15-17, 2017, Crowne Plaza Manila; at the Common Pediatric GI Problems: Revisited $12^{\text {th }}$ Annual Convention Philippine Society for Pediatric Gastroenterology, Hepatology and Nutrition, May 2-3, 2017, Crowne Plaza Manila; and at the $33^{r d}$ Annual Pediatric Research Week, November 2017, Philippine General Hospital (Third place, Fellows' Research Paper Contest).

Corresponding author: Germana Emerita V. Gregorio, MD, PhD Department of Pediatrics Philippine General Hospital University of the Philippines Manila Taft Avenue, Manila 1000, Philippines

Email: gvgregorio@up.edu.ph
Tuberculosis (TB), which is caused by Mycobacterium tuberculosis, is a global health problem with about nine million new cases reported annually. According to the National Tuberculosis Control Program (NTP), 1,379,390 cases were reported in the Philippines from 2003 to 2011,1.1\% of which were extra-pulmonary TB. ${ }^{1}$ In children, the actual burden of childhood TB is unknown but the estimated number of TB cases was 530,000 in 2012. ${ }^{2}$ Tuberculosis may cause liver involvement in three ways: (1) miliary dissemination of the tubercle bacilli via the lymphatic channels or the bloodstream; (2) granulomatous or tuberculous inflammation of the liver; or (3) hepatobiliary tuberculosis with bile duct lesions due to the invasion of periportal lymph nodes or involvement of the ductal epithelium. ${ }^{3}$ 
There are several reported cases of hepatobiliary tuberculosis from China, Korea, Taiwan, South Africa, India and the Philippines, mostly among adults from 19 to 75 years old. In several case series of children, 48 to $60 \%$ of cases present with abdominal pain and 85 to $96 \%$ have findings of hepatomegaly. Jaundice was only observed in $10 \%$ of patients. ${ }^{4,5}$ Liver enzyme abnormalities were common but these were not considered diagnostic of hepatobiliary TB. In all the studies, abdominal CT scan is the most sensitive imaging modality (88\%) while the most specific diagnostic test is performing a liver biopsy (96\%). ${ }^{6}$ Histopathologic findings in the liver of epithelioid cell granuloma and caseation necrosis, which result from the organization of lymphocytes, macrophages, Langhans giant cells and fibroblasts, confirm the diagnosis of hepatobiliary tuberculosis. Culture of TB bacilli is also diagnostic but the sensitivity is only $40-50 \%$ due to the difficulty of specimen collection and paucibacillary nature of the disease. The detection of acid fast bacilli (AFB) in microscopically examined stained smears provides a presumptive diagnosis of active tuberculosis and the quantitative estimation of the number of bacilli indicates the degree of infectivity of the patient. ${ }^{7}$

The features and outcome of hepatobiliary tuberculosis have not been extensively reported in the pediatric population. ${ }^{4}$ In children, signs and symptoms of hepatic tuberculosis such as jaundice, fever and hepatomegaly, are also seen in other chronic liver diseases. ${ }^{5}$ Histological confirmation entails obtaining a liver biopsy specimen which is invasive ${ }^{6}$ and may not always be possible in suspected cases due to deranged bleeding parameters as a result of the liver pathology. Caseation necrosis in the liver, the hallmark of tuberculosis, is also rarely seen. It is best to have a bacteriologically-confirmed diagnosis with a positive AFB smear or mycobacterial culture from an extra-pulmonary site such as CSF, urine, pleural, pericardial or joint fluid. However, the sensitivity of both the AFB smear and culture is only from 20 to $50 \%$. A nucleic acid amplification test may be necessary, though its use in our setting is limited by the prohibitive cost. ${ }^{7}$ Most patients therefore are only diagnosed by their response to empiric anti-tuberculosis drug regimen. This therapeutic approach is dangerous as it exposes a patient without the disease to the adverse effects of the drug, foremost of which is hepatotoxicity.

The objective of this study was to describe the clinical, biochemical, microbiologic, radiologic and histologic features and outcome of pediatric patients diagnosed with hepatobiliary tuberculosis from 2010 to 2015 .

\section{METHODS}

\section{Study design and setting}

This was both a retrospective and a prospective cohort study conducted at the Section of Pediatric Gastroenterology, Hepatology and Nutrition of the University of the Philippines Manila's Philippine General Hospital, a government tertiary referral center in the country.

\section{Subjects investigated}

All patients diagnosed with hepatobiliary tuberculosis were included. A diagnosis of hepatic tuberculosis was made on any patient with clinical, biochemical, radiologic or histologic evidence of liver disease and was identified as bacteriologically-confirmed or clinically-diagnosed. Bacteriologically-confirmed cases are those whose liver tissue sample is positive on acid fast bacilli smear, culture or polymerase chain reaction (PCR) examination. Clinicallydiagnosed cases are those with clinical, histologic and/or radiologic evidence consistent with active extra-pulmonary tuberculosis and decision of the attending physician to treat with anti-tuberculosis drugs. These may consist of at least one of the following: (1) histologic evidence of caseating granuloma and/or Langhans multinucleated giant cell in the liver; (2) abdominal ultrasound or CT scan demonstrating low density hepatic nodules in patients with confirmed pulmonary TB; or (3) clinical improvement following anti-TB treatment including resolution of fever, reduction of jaundice, improvement of appetite or decrease in the size of the liver. ${ }^{6,8}$

For the retrospective part (2010-2014), consecutive patients 0-18 years with the final diagnosis of hepatobiliary tuberculosis were identified based on the census of both the Section of Pediatric Gastroenterology, Hepatology, and Nutrition and the Section of Pediatric Infectious Diseases and Tropical Medicine. The medical charts of these patients were retrieved and relevant information were recorded on the patient data sheet.

For the prospective part (2015), all patients with a diagnosis of hepatobiliary tuberculosis were included. The purpose and mechanics of the study were discussed with the parent or guardian and if possible, with the candidate subject. Informed consent from the parent or guardian and assent from the candidate subjects $>12$ years old was obtained. Complete history and physical examination were done by the principal investigator and all information significant to the study were recorded.

\section{Parameters investigated}

In all patients, pertinent clinical, biochemical, microbiologic and radiologic features and outcome were obtained. These included whether the patient had BCG immunization, previous diagnosis of primary complex or PTB and exposure to any member of the household diagnosed with tuberculosis. Information on the presence or absence of fever, weight loss, anorexia, abdominal pain, abdominal distension, jaundice, acholic stools and teacolored urine were noted. Pertinent findings on physical examination were documented including the nutritional status, anthropometric measurements and presence or absence of jaundice, hepatomegaly, splenomegaly, ascites, spider angioma and palmar erythema. 


\section{Biochemical and Microbiologic Findings}

The initial results of the complete blood count, prothrombin time (reported as international normalized ratio or INR) and liver function tests including total and direct bilirubin, aspartate and alanine aminotransferases, alkaline phosphatase and albumin were obtained. A positive finding of any biologic specimen sent for $\mathrm{AFB}$ smear and TB culture studies were noted. If the TB culture is positive, the sensitivity patterns to various anti-TB drugs were logged.

\section{Imaging Studies}

Chest $\mathrm{x}$-ray results of pulmonary tuberculosis such as lymphadenopathy, calcification, consolidation, atelectasis and pleurisy were documented ${ }^{7}$. Findings on abdominal ultrasound, computerized tomography scan and/or magnetic resonance imaging of presence of hepatomegaly, splenomegaly, ascites, calcifications, lymphadenopathies, bile duct dilatation and nodularities were recorded.

\section{Histopathologic Findings}

The histopathology reports of the liver biopsy of patients were reviewed as to the presence of caseating granuloma, inflammation, and Langhans multinucleated giant cells.

\section{Outcome}

The duration of follow up was recorded from initial diagnosis to date of last follow up. In the retrospective cohort, the outcome was assessed based on the recorded clinical course during hospital confinement and the outpatient clinic follow up. For all patients both in the retrospective and prospective cohort, they were followed up for at least six months from initial diagnosis or until the time of last known hospital visit.

The medications given, duration of intake and adverse drug events were documented such as nausea, vomiting, abdominal pain, jaundice and elevation of serum liver enzymes. ${ }^{7}$ Any surgical procedure performed on a patient and the intra-operative findings were recorded. The outcome of the patient was described as alive (with or without sequelae), died or lost to follow-up. Sequelae as a result of hepatic tuberculosis, such as obstructive jaundice, liver failure or development of cirrhosis were also noted. Patient was considered lost to follow up if there was no consult for at least six months from time of first visit.

The research was approved by the Ethics Review Board of the Philippine General Hospital.

\section{RESULTS}

During the six-year study period, 36 patients were diagnosed to have hepatobiliary tuberculosis, accounting for $5.4 \%$ of all diagnosed tuberculosis cases in children at our institution. Three cases were bacteriologically-confirmed on the basis of positive TB culture of the bile (two cases) and TB PCR of the liver tissue (one). One of these patients also had histologic findings of hepatobiliary TB. Thirty-three were clinically-diagnosed: eight had histologic evidence from liver biopsy, 12 with radiographic findings, and 13 subjects based solely on clinical resolution after anti-TB treatment. Information on 33 patients were obtained by chart review while data was prospectively collected on three patients during hospital admission.

\section{Clinical Features (Table 1)}

The mean age at diagnosis was thirteen years old, with the youngest at six months and the oldest at eighteen years old. The majority (83\%) of patients were between 11 to 18 years of age. Male predominance (64\%) was observed.

There were 31 patients (86\%) who were immunized with Bacilli Calmet-Guerrin (BCG) vaccine based on history or note of BCG scar. Twenty-two subjects (61\%) were exposed to persons with tuberculosis but only seven (19\%) had pulmonary tuberculosis before being diagnosed with hepatobiliary tuberculosis. Of the seven, only three completed six months of anti-TB treatment.

The mean duration of illness before diagnosis of hepatobiliary tuberculosis was 48 weeks, from as early as 3 weeks to as late as 6 years from onset of symptoms. The most common clinical feature was weight loss (69\%). Nonspecific symptoms like fever (67\%), abdominal pain (56\%), abdominal distention (53\%) and anorexia (31\%) were also observed. Signs of an obstructive pathology, including

Table 1. Demographic and clinical profile of 36 patients diagnosed with hepatobiliary tuberculosis, n (\%)

\begin{tabular}{lc}
\hline Age (years) & $\mathbf{n}(\%)$ \\
$<5$ & $1(3)$ \\
$5-10$ & $5(14)$ \\
$11-14$ & $14(39)$ \\
$15-18$ & $16(44)$ \\
\hline Sex & \\
Male & $23(64)$ \\
Female & $13(36)$ \\
\hline History of TB exposure & \\
Yes & $22(61)$ \\
No & $14(39)$ \\
\hline BCG vaccination & \\
Yes & $31(86)$ \\
No & $5(14)$ \\
\hline Clinical presentation & \\
Weight loss & $25(69)$ \\
Fever & $24(67)$ \\
Jaundice & $22(61)$ \\
Abdominal pain & $20(56)$ \\
Abdominal distention & $19(53)$ \\
Anorexia & $11(31)$ \\
Tea-colored urine & $4(11)$ \\
Acholic stools & $1(3)$ \\
\hline
\end{tabular}


jaundice (61\%), tea-colored urine (11\%) and acholic stools (3\%) were reported. On physical examination, there was note of hepatomegaly (61\%), jaundice (53\%), ascites (42\%) and splenomegaly (31\%).

Of the 36 patients, only six (16\%) had isolated hepatobiliary tuberculosis. Thirty patients (84\%) had disseminated TB with liver involvement, three of whom had no lung lesion but with TB meningitis, pancreatic TB or ileocecal TB (Table 2).

\section{Biochemical and Microbiologic Findings (Table 3)}

There were twenty-nine patients (81\%) with anemia (hemoglobin < $120 \mathrm{~g} / \mathrm{L}$ ) and eighteen (50\%) had leukocytosis (WBC count $>11 \times 10^{9} / \mathrm{L}$ ). An increase in alanine transferase, aspartate transferase, alkaline phosphatase and bilirubin levels were observed in 18 (50\%), 19 (53\%), 33

Table 2. Organ involvement of 36 patients diagnosed with hepatobiliary tuberculosis, n (\%)

\begin{tabular}{lc}
\multicolumn{1}{c}{ Diagnosis } & $\mathbf{n}(\%)$ \\
Isolated hepatobiliary TB & $6(16.7 \%)$ \\
Disseminated TB* & $30(83.3 \%)$ \\
Other organs involved: & \\
Lungs & 27 \\
Lymph node & 7 \\
Pancreas & 7 \\
Gastrointestinal tract & 6 \\
Heart & 5 \\
Kidney & 3 \\
Spine & 3 \\
Spleen & 3 \\
Uterus & 2 \\
Brain & 1 \\
\hline
\end{tabular}

* Some patients had two or more organs involved apart from the liver

Table 3. Mean and standard deviation of the initial laboratory investigations of 36 patients diagnosed with hepatobiliary tuberculosis

\begin{tabular}{|c|c|c|c|}
\hline & $\begin{array}{l}\text { No. of } \\
\text { Patients }\end{array}$ & Normal Values & Mean \pm SD \\
\hline CBC & 36 & & \\
\hline Hemoglobin & & $120-160 \mathrm{~g} / \mathrm{L}$ & $97.69 \pm 33.12$ \\
\hline Platelet & & $150-450 \times 10^{9} / \mathrm{L}$ & $315.61 \pm 153.28$ \\
\hline WBC & & $4.5-11 \times 10^{9} / \mathrm{L}$ & $12.14 \pm 6.56$ \\
\hline Neutrophils & & $0.50-0.70$ & $0.71 \pm 0.14$ \\
\hline Lymphocytes & & $0.20-0.50$ & $0.18 \pm 0.11$ \\
\hline AST & 36 & $22-58 \mathrm{IU} / \mathrm{L}$ & $79.67 \pm 70.31$ \\
\hline ALT & 36 & $11-39$ IU/L & $60.33 \pm 57.35$ \\
\hline Total bilirubin & 35 & $0-2 \mathrm{mg} / \mathrm{Dl}$ & $4.50 \pm 5.86$ \\
\hline Direct bilirubin & 35 & 0-0.9 mg/DI & $2.89 \pm 3.65$ \\
\hline Alkaline Phosphatase & 23 & $32-91 \mathrm{IU} / \mathrm{L}$ & $494.65 \pm 353.94$ \\
\hline Albumin & 31 & $31-48 \mathrm{~g} / \mathrm{L}$ & $24.33 \pm 10.14$ \\
\hline Glucose & 30 & $4.38-6.38 \mathrm{mg} / \mathrm{dL}$ & $5.84 \pm 4.62$ \\
\hline INR & 36 & $0.8-1.2$ & $1.54 \pm 1.08$ \\
\hline
\end{tabular}

(91\%) and 14 (40\%) patients, respectively. Majority (68\%) had hypoalbuminemia (serum albumin of less than $31 \mathrm{~g} / \mathrm{L}$ ) and $47 \%$ had deranged prothrombin time (INR >1.2).

Overall, 15 of the 36 patients yielded positive on either AFB smear, TB culture and/or TB PCR (Table 4). The three samples that were positive on TB PCR were from the pleural fluid, sputum and a liver biopsy specimen. Of the 16 specimens that were positive on TB culture from 9 patients, 14 samples had resistance to at least one drug including: isoniazid (11), pyrazinamide (10), rifampicin (8), ethambutol (7) and streptomycin (7).

\section{Imaging Studies}

Out of the 32 patients whose chest radiography results were reviewed, five (15\%) had normal results. The chest $\mathrm{x}$-ray of twenty-seven patients had findings of opacity (37\%), pleural effusion (19\%), consolidation (9\%), cavity formation (9\%), atelectasis (9\%), apical infiltrates (9\%), nodularity (9\%) and lymphadenopathy (6\%). Only one (3\%) had pulmonary calcification.

Majority of the patients had hepatic calcification (64\%) and hepatosplenomegaly (53\%) which was seen on either the US, CT scan and/or MRI. Other findings that were commonly seen included lymphadenopathy at the porta hepatis (47\%), dilated bile ducts (47\%) and hepatic nodularity (44\%). (Table 5) (Figure 1)

\section{Histopathologic Findings (Figure 2)}

Of 11 patients who underwent liver biopsy, seven had chronic or granulomatous inflammation (two with Langhans multinucleated giant cell also), one with Langhans multinucleated giant cell only, another with tuberculous caseation and two with cirrhosis with no evidence of hepatobiliary tuberculosis, one of whom was positive on TB PCR.

Table 4. Result of biologic specimen that were positive for AFB stain, TB culture and TB PCR among the 36 patients diagnosed with hepatobiliary tuberculosis*

\begin{tabular}{lccc}
\multicolumn{1}{c}{ Specimen } & AFB stain & TB Culture & TB PCR \\
Pulmonary & & & \\
$\quad$ Sputum & $7 / 30$ & $6 / 12$ & $1 / 2$ \\
$\quad$ Gastric aspirate & $3 / 11$ & $2 / 5$ & $0 / 1$ \\
\hline Extrapulmonary & & & \\
$\quad$ Bile & $0 / 1$ & $2 / 2$ & $0 / 1$ \\
Liver tissue & $0 / 1$ & - & $1 / 3$ \\
Urine & $3 / 28$ & $1 / 12$ & - \\
Stool & $5 / 10$ & $2 / 4$ & - \\
Pleural fluid & $0 / 1$ & - & $1 / 2$ \\
Pericardial fluid & $1 / 1$ & - & - \\
Peritoneal fluid & - & $1 / 1$ & - \\
Wound Aspirate/Discharge & - & $2 / 3$ & - \\
\hline Total, $\mathrm{n}$ (\%) & $19 / 83(23 \%)$ & $16 / 39(41 \%)$ & $3 / 9(33 \%)$ \\
\hline * Patients may have more than (n) & bilogic specimen submitted
\end{tabular}

* Patients may have more than one biologic specimen submitted and more than one examination done 


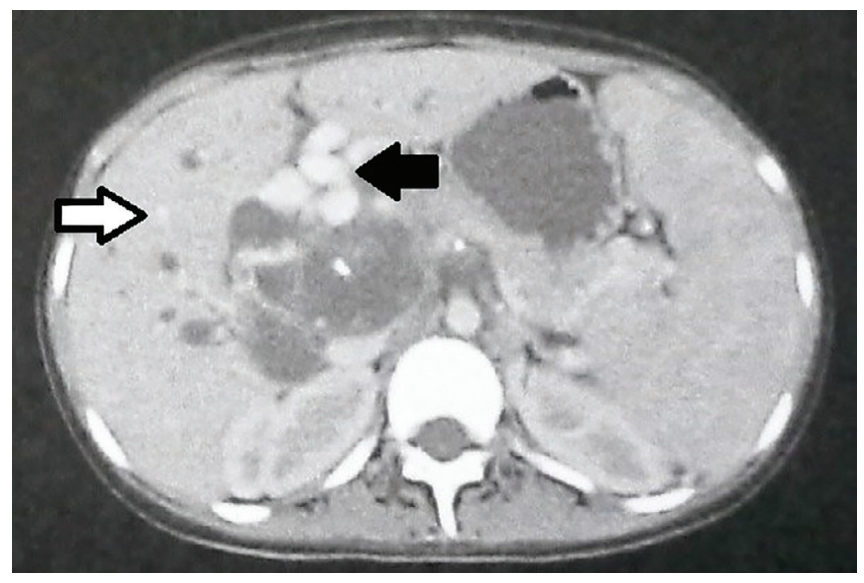

Figure 1. Abdominal CT scan of a 15-year-old female with calcific densities in the right liver lobe (white arrow) and confluent periportal lymph nodes (black arrow).

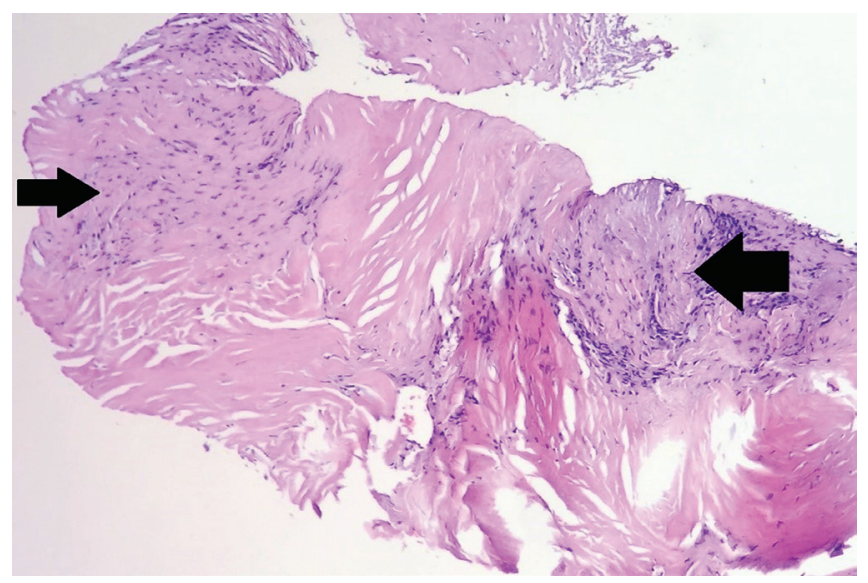

Figure 2. Histologic findings of tuberculous caseation (black arrow) [hematoxylin eosin stain 40X] from a liver biopsy specimen of a 13-year-old male.

Table 5. Imaging findings at presentation of 36 patients diagnosed with hepatobiliary tuberculosis*

\begin{tabular}{lcccc} 
& $\begin{array}{c}\text { Ultrasound } \\
(\mathbf{N}=\mathbf{3 0})\end{array}$ & $\begin{array}{c}\text { Abdominal CT Scan } \\
(\mathbf{N}=\mathbf{2 5})\end{array}$ & $\begin{array}{c}\text { MRI of the abdomen } \\
(\mathbf{N}=5)\end{array}$ & $\begin{array}{c}\text { Patients with this finding, } \\
\mathbf{n}(\%)\end{array}$ \\
Hepatomegaly & 14 & 11 & 2 & $19(53)$ \\
Splenomegaly & 12 & 13 & 4 & $20(56)$ \\
Dilated bile ducts & 11 & 9 & 3 & $17(47)$ \\
Ascites & 10 & 4 & 1 & $15(42)$ \\
Hepatic calcification & 9 & 17 & 2 & $23(64)$ \\
Hepatic nodularity & 6 & 10 & - & $16(44)$ \\
Hepatic mass & 4 & 3 & 2 & $6(17)$ \\
Lymphadenopathy at the porta hepatis & 3 & 14 & 2 & $17(47)$ \\
Prominent portal vein & 3 & 6 & 2 & $10(28)$ \\
Beaded appearance of biliary tree & 1 & - & - & $2(6)$ \\
Collateral vessel formation & 1 & 4 & & $4(11)$ \\
\hline
\end{tabular}

* 19 patients had both ultrasound or CT scan of the abdomen

\section{Treatment}

All patients were managed medically. Six also had endoscopic or surgical intervention. Thirty-two subjects were given quadruple therapy (isoniazid, rifampicin, pyrazinamide, ethambutol). In addition, streptomycin was administered to four children due to previous incomplete anti-TB treatment.

For nine patients with resistance to at least one antituberculosis drug on TB culture, eight were still treated with the first line drug regimen while one was given ofloxacin in addition to quadruple treatment due to endobronchial TB. Drug resistance to isoniazid was observed in seven patients, rifampicin in six, pyrazinamide in eight and ethambutol in six. Seven patients had more than one drug resistance.

Five of the 36 patients developed adverse drug reactions to treatment including two times elevation of transaminases (100\%), jaundice (60\%), abdominal pain (40\%) and vomiting (20\%). Four of the five responded with re-challenge of the first line drugs at reduced dose and in one, rifampicin was stopped and was replaced with levofloxacin.

Endoscopic rubber band ligation was performed in two patients for bleeding esophageal varices. A 12-year-old male had hepatic resection for liver abscess and an endoscopic retrograde cholangiopancreatography, sphincterotomy and stent placement to provide adequate bile flow. Two patients had percutaneous transhepatic biliary drainage for ascending cholangitis and one had cholecystectomy and hepaticoduodenostomy for common bile duct stricture.

\section{Outcome (Table 6)}

Of the 36 patients, 22 patients were alive, eight were lost to follow up and six have died. The 22 living patients were followed up from 6 to 52 months [Mean (SD): 18 (13) months] from diagnosis wherein 19 completed six to 12 months of anti-TB treatment while there was no record whether the three others complied with treatment. Only nine of the 22 had no signs and symptoms of liver disease while 13 others developed liver related complications. 
Table 6. Outcomes of 36 patients diagnosed with hepatobiliary tuberculosis, $\mathrm{n}(\%)$

\begin{tabular}{lc}
\multicolumn{1}{c}{ Outcome } & $\mathbf{n}(\%)$ \\
\hline Alive & $22(61.1 \%)$ \\
Without sequelae & 9 \\
With sequelae* & 13 \\
Obstructive jaundice & 8 \\
Portal hypertension & 6 \\
Sclerosing cholangitis & 1 \\
Died & $6(16.7 \%)$ \\
Lost to follow up & $8(22.2 \%)$ \\
\hline
\end{tabular}

* Some patients have two or more complications

Six patients died after two to 93 days of admission. The causes of death were hypovolemic shock from massive upper gastrointestinal bleeding in four and disseminated intravascular coagulation secondary to sepsis in two cases.

\section{DISCUSSION}

There are limited reports of hepatobiliary tuberculosis in children, accounting for 11 of 96 histologically diagnosed patients in South Africa ${ }^{5}$, two of seven cases from India ${ }^{9}$ and 40 of 120 patients from the Philippines ${ }^{4}$. All these studies reported their findings together on both adults and children, thus it is difficult to identify the features that characterize only the pediatric age group. This study provides novel information on the mode of presentation, diagnostic tools and prognosis of hepatobiliary tuberculosis in children.

Our research showed that hepatobiliary tuberculosis is uncommon and often presents with non-specific clinical and biochemical findings. It accounted for $5.4 \%$ of all tuberculosis cases admitted in the institution during the study period which is higher than the $1.2 \%$ of diagnosed TB patients at a hospital in South Africa. ${ }^{10}$ The Philippine General Hospital is a tertiary medical center where patients with more severe liver conditions are referred. In 2010, tuberculosis was the sixth leading cause of death (26.3 deaths $/ 100,000$ population $)^{8}$ in the country and was the third highest cause of mortality among immunizable diseases. ${ }^{11}$

Fever and weight loss were the most common presenting symptoms seen in $67 \%$ and $69 \%$ of our cases similar to the 65 to $100 \%$ observed in earlier reports. ${ }^{4,12}$ In terms of physical findings, more than half of our patients had hepatomegaly (61\%) and jaundice (53\%). Previous studies in adults have noted hepatomegaly from 80 to $95 \%$ but only 10 to $20 \%$ had jaundice. ${ }^{5,9,12}$

Due to the non-specific symptoms of the disease, majority of our patients were diagnosed late with a mean duration of 48 weeks from initial symptoms. The longer the illness, the greater will be the inflammation and destruction of the hepatocytes or obstruction of the bile ducts by lymph nodes leading to jaundice. On the other hand, hepatomegaly maybe secondary to a hepatic mass, nodular formation or inflammation of hepatocytes. Corollary to these is our findings of increased levels of bilirubin (40\%) and transaminases (50\%), deranged prothrombin time (47\%) and low albumin $(68 \%)$, the latter two denoting poor synthetic function of the liver suggestive of severe liver disease.

The findings on routine chest $\mathrm{x}$-ray of opacity, pleural effusion, cavity formation or calcification may aid in the diagnosis of hepatobiliary tuberculosis. However, a normal chest radiograph does not rule out the diagnosis as we have observed in five of $32(16 \%)$ patients and in 25 to $35 \%$ of cases in previous reports. ${ }^{4,5}$ Abdominal imaging studies are employed as important parameters to make a diagnosis of hepatobiliary tuberculosis. Both ultrasound of the liver and contrast-enhanced abdominal CT scan are used to document the presence of liver nodules which was noted in $44 \%$ of our patients but seen in 10 of 12 (83\%) cases in China. ${ }^{13}$ Other findings that may lead to a suspicion of hepatobiliary tuberculosis are the presence of calcification (64\%) and lymphadenopathy at the porta hepatis (47\%) which were mostly observed in our patients but only reported in $29 \%$ and $8 \%$ of cases, respectively in previous studies. ${ }^{12,13}$ Hepatic calcification indicates that calcium has been formed in areas of the liver that were affected by injury. If on screening with hepatobiliary ultrasound the presence of biliary dilatation is noted, a magnetic resonance cholangiopancreaticography (MRCP) is more sensitive than CT scan in demarcating biliary anatomy. Two of five of our patients with an MRCP had the characteristic beaded appearance of the biliary tree, suggestive of sclerosing cholangitis, a sequela of hepatobiliary tuberculosis. ${ }^{14,15}$

In patients suspected of hepatic tuberculosis, microbiologic studies of specimen from pulmonary and extrapulmonary sources are requested to detect the presence of the bacilli through an AFB smear and if possible for a $\mathrm{TB}$ polymerase chain reaction and culture and sensitivity studies. Using auramine and Ziehl-Neelsen staining, only $23 \%$ of our submitted specimens were positive on AFB stain consistent with the expected sensitivity of around 20\%. Culture of Mycobacterium tuberculosis was positive in $41 \%$ in our study, two of which were bile specimens with resistance to at least one of the first line drugs. No liver tissue was sent for culture but three were submitted for PCR, one of which was positive. In a systematic review, positive AFB stain on aspiration and of culture of liver tissue of hepatic tuberculosis patients has a median sensitivity of $25 \%$ and $<10 \%$, respectively. ${ }^{6}$

All our patients were treated based on the guidelines of the National Tuberculosis Control Program with consideration of their drug susceptibility pattern. Twenty five percent (nine of 36) of our patients had complete recovery after treatment without signs and symptoms of liver disease which is higher than the $11 \%$ reported by Alvarez in predominantly adult cases. ${ }^{4}$ However, the use of multi-drug therapy resulted in adverse drug reactions in five (14\%) patients including at least two times increase in the transaminase value and elevation 
Hepatobiliary Tuberculosis among Filipino Children

in bilirubin levels. Hepatotoxicity attributed to anti-TB drug has been reported from five to $28 \%$ of cases worldwide and in the presence of liver disease, the likelihood of druginduced hepatitis is higher. In 107 patients with chronic liver disease, independent risk factors for drug-induced liver injury included female gender, number of hepatotoxic drugs administered and baseline alkaline phosphatase levels. ${ }^{16}$ Anti-tuberculosis drug-induced fulminant liver failure has also been reported. ${ }^{17}$ Although resistance to at least one antituberculosis drug was noted in nine patients in our study, eight were still treated using first-line anti-TB medications as the culture results were only available after two months and the patients were already responding to treatment. However, this is not recommended since poor drug regimen involving weak combination of drugs or using those with low bioavailability further amplifies drug resistance ${ }^{18}$ which might lead to dismal outcome.

For the 13 other patients who are still alive with sequelae, treatment was mainly directed to the complication of chronic liver disease including decreasing jaundice and pruritus with choleretics, prevention of bleeding with use of beta-blocker and endoscopic treatment, relief of ascites and nutritional support. Should their liver status further deteriorate, these patients will be a candidate for a liver transplant after it is established that they have been cured of tuberculosis. Presently, review of literature showed that no patient yet has undergone liver transplant for end stage liver disease secondary to hepatobiliary tuberculosis.

\section{CONCLUSION}

The present study showed that hepatobiliary tuberculosis is uncommon and often presents with non-specific clinical and biochemical findings which can mimic other liver diseases. Once the diagnosis is suspected, microbiologic, radiologic and/or histologic investigations are essential to confirm the diagnosis. Overall response to anti-TB treatment is satisfactory with complete resolution of symptoms in some patients.

\section{Recommendation}

The present study was conducted in a single institution and is limited by information mostly obtained from chart review. A multi-center survey is recommended with adequate microbiologic, radiologic and if possible, histologic investigations performed in all patients suspected of having hepatobiliary tuberculosis. Serial biochemical monitoring should also be carried out while on anti-tuberculosis treatment and the outcome of patients should be assessed.

\section{Statement of Authorship}

All authors participated in data collection and analysis, and approved the final version submitted.

\section{Author Disclosure}

All authors declared no conflicts of interest.

\section{Funding Source}

This paper was funded by the Pediatric Infectious Disease Society of the Philippines (PIDSP).

\section{REFERENCES}

1. Vianzon R, Garfin A., Lagos A, Belen R. The tuberculosis profile of the Philippines, 2003-2011: advancing DOTS and beyond. Western Pac Surveill Response J. 2013 Apr-Jun; 4(2):11-6.

2. World Health Organization. Guidance for national tuberculosis programmes on the management of tuberculosis in children. Geneva: WHO Document Production Services. 2014. p. 13.

3. Chaudhary P. Hepatobiliary tuberculosis. Ann Gastroenterol. 2014; 27(3):207-11.

4. Alvarez SZ, Carpio R. Hepatobiliary tuberculosis. Dig Dis Sci. 1983 Mar; 28(3): 193-200.

5. Essop AR, Posen JA, Hodkinson JH, Segal I. Tuberculosis hepatitis: a clinical review of 96 cases. QJ Med. 1984; 53(212) 465-77.

6. Hickey AJ, Gounder L, Moosa MYS, Drain PK. A systematic review of hepatic tuberculosis with considerations in human immunodeficiency virus co-infection. BMC Infect Dis. 2015 May; 15:209.

7. Philippine Pediatric Society, Inc. Tuberculosis in Infancy and Childhood. Quezon City; 2010.

8. Department of Health. National TB Control Program Manual of Procedures, fifth edition. Manila: Department of Health; 2014. pp. 18-20.

9. Desai CS, Joshi AG, Abraham P, Desai DC, Deshpande RB, Bhaduri A, et. al. Hepatic tuberculosis in the absence of disseminated abdominal tuberculosis. Ann Hepatol. 2006 Jan-Mar; 5(1):41-3.

10. Azad K, Mallik MU, Hossain MZ, Shayekh A, Alam A, Sarker P. Isolated hepatic tuberculosis. J Dhaka Med Coll. 2015; 23(1):121-3.

11. DOH. Philippine Health Statistics 2001-2010 Mortality among immunizable diseases [Internet]. May 5, 2014 [cited 2015 April]. Available from: http://www.doh.gov.ph/Statistics/Mortality-AmongImmunizable Diseases.

12. Vilaichone RK, Mahachai V. Hepatic tuberculosis: a clinicopathological study. Thai J Gastroenterol. 2004; 5(1):19-23.

13. Yu RS, Zhang SZ, Wu JJ, Li RF. Imaging diagnosis of 12 patients with hepatic tuberculosis. World J Gastroenterol. 2004 Jun; 10(11): 1639-42.

14. Alvarez SZ. Hepatobiliary Tuberculosis. Phil J Gastroenterol. 2006; 2:1-10.

15. Jain A, Chaturvedi R, Kantharia C, Joshi A, Londhe M, Kekan M. Secondary sclerosing cholangitis in localized hepatobiliary tuberculosis simulating cholangiocarcinoma: a rare case report. BMC Gastroenterol. 2017 Nov; 17(1):126.

16. Park WB, Kim W, Lee KL, Yim JJ, Kim M, Jung YJ, et. al. Antituberculosis drug-induced liver injury in chronic hepatitis and cirrhosis. J Infect. 2010 Oct; 61(4):323-9.

17. Kumar R, Shalimar Bhatia V, Khanal S, Sreenivas V, Datta Gupta S, Panda SK, et al. Antituberculosis therapy-induced acute liver failure: magnitude, profile, prognosis, and predictors of outcome. Hepatology. 2010 May; 51(5):1665-74.

18. Schaaf HS, Marais BJ. Management of multidrug-resistant tuberculosis in children: A survival guide for paediatricians. Paediatr Respir Rev. 2011 Mar; 12(1):31-8. 